\title{
INVERSION OF THREE LAYERS MULTI-SCALE SPM MODEL BASED ON NEURAL NETWORK TECHNIQUE FOR THE RETRIEVAL OF SOIL MULTI-SCALE ROUGHNESS AND MOISTURE PARAMETERS
}

\author{
I. Hosni $*^{\mathrm{a}}$ M. JaafriGhamki, ${ }^{\text {ab }}$, L. Bennaceur Farah ${ }^{\mathrm{a}}$ and M. S. Naceur ${ }^{*^{\mathrm{a}}}$ \\ a'LTSIRS, ENIT, Université El Manar, Tunis, Tunisia - hosni_ibtissem@yahoo.fr; lilia.bennaceur@ipeit.rnu.tn \\ ${ }^{\mathrm{b}}$ RIADI, ENSI, Université de la Manouba, Tunis, Tunisia - jaafrimaroua@yahoo.fr
}

KEY WORDS: Backscattering, multi-scale, multilayer, inversion, neural network

\begin{abstract}
:
In this paper, a multi-layered multi-scale backscattering model for a lossy medium and a neural network inversion procedure has been presented.

We have used a bi-dimensional multi-scale (2D MLS) roughness description where the surface is considered as a superposition of a finite number of one-dimensional Gaussian processes each one having a spatial scale using the wavelet transform and the Mallat algorithm to describe natural surface roughness.

An adapted three layers 2D MLS small perturbations (SPM) model has been used to describe radar backscattering response of semiarid sub-surfaces. The total reflection coefficients of the natural soil are computed using the multilayer model, and volumetric scattering is approximated by the internal reflections between layers. The original multi-scale SPM model includes only the surface scattering of the natural bare soil, while the multilayer soil modified 2D MLS SPM model includes both the surface scattering and the volumetric scattering within the soil. This multi-layered model has been used to calculate the total surface reflection coefficients of a natural soil surface for both horizontal and vertical co-polarizations.
\end{abstract}

A parametric analysis presents the dependence of the backscattering coefficient on multi scale roughness and soil.

The overall objective of this work is to retrieve soil surfaces parameters namely roughness and soil moisture related to the dielectric constant by inverting the radar backscattered signal from natural soil surfaces.

To perform the inversion of the modified three layers 2D MLS SPM model, we used a multilayer neural network (NN) architecture trained by a back-propagation learning rule.

\section{INTRODUCTION}

Over the last two decades, microwave remote sensing has become an efficient tool for indirectly estimating soil moisture and soil properties in the top few centimeters of soils at different spatial and temporal scales. Soil moisture affects the partitioning of rainfall into infiltration and runoff and modulates soilatmosphere feedback interactions and it also affects groundwater recharge, crop growth.

In that context, modeling radar backscattering through natural surfaces has become an important theme of research and active remote sensing and has shown its utility for many applications in hydrology, geology, astrophysics, etc

The characterization of soil surface roughness is a key requirement for the correct analysis of radar backscattering behavior. Many previous works have been devoted to the analysis of the backscattering characteristics of bare soils and several backscattering models (theoretical, semi- empirical and empirical) were developed ([1] [2] [6] [9]). They used the classical statistical description of natural surfaces and characterized roughness by statistical parameters namely correlation length and standard deviation.

However, the weakness of the classical description of natural surfaces is the large spatial variability which affects the correlation function and makes classical roughness parameters very variable. Several works have proposed various approaches for the improvement of roughness descriptions ([3] [4] [5] [7]
[11]) and have suggested that natural surfaces are better described as self-affine random processes (1/f processes) than as stationary processes. In previous works, we have analyzed radar backscattering on multi-scale bi-dimensional surfaces [3][7][10] which description does not depend on classical roughness parameters standard deviation and correlation length but on new parameters related to multi-scale surfaces properties.

Extracting soil moisture and roughness parameters of natural surfaces from this data has been problematic for many reasons and many researchers have encountered many problems like the lack of information about the characteristics of natural surface roughness. In addition, the relation-ship between the backscattering coefficients is non-linear and the problem of retrieving parameters is frequently ill-posed and it may be impossible to separate the contributions from different mechanisms making the retrieval of several parameters simultaneously necessary.

The objective of this paper is to develop and test an inversion algorithm for soil moisture and multi-scale roughness parameters retrieval from radar backscattering coefficients simulated by the modified SPM model using a neural network inversion procedure based on a multilayer neural network (NN) architecture trained by a back propagation learning rule.

This paper is organized into five sections. The first section describes the two dimensional multi-scale description of natural rough surfaces. Section 2 presents the multi-layers MLS SPM model. The third section discusses the influence of multi-scale roughness and the dielectric constant related to soil moisture on the backscattering simulations using our three layers multi-scale 
bi-dimensional small perturbation model (SPM). In the next section the neural network based inversion procedure, the results and their accuracy are presented. Finally, our conclusions are presented in the last section.

\section{A MULTISCALE DESCRIPTION OF NATURAL SOILS ROUGHNESS}

In this section, we present the multi-scale surface model used in the SPM model.

Natural roughness is described as a multi-scale process having a $1 / f$ spectrum with a finite range of spatial scales going from a few millimeters $\left.\mathrm{b}\left(b \leq \frac{\lambda}{10}\right)\right)$ to several meters ( $\mathrm{B} \leq$ resolution cell) [3][7]. We have considered the surface as a superposition of a finite number of one-dimensional Gaussian processes each one having a spatial scale [1] characterized by:

$$
Z_{p}(x)=\sum_{m=-P_{1}}^{P_{2}} \sum_{n=-\infty}^{+\infty} Z_{n}^{m} \Psi_{n}^{m}\left(\frac{x}{L}\right)
$$

Where $Z_{n}^{m}$ is a collection of gaussian random independent variables with variance $\gamma_{0}^{2} 2^{-m}, \mathrm{x}$ a normalized distance with respect to an arbitrary length $\mathrm{L}=2^{\mathrm{b}} \mathrm{b}$ and $\Psi_{n}^{m}$ a collection of orthonormal wavelet (4th Daubechies). The roughness multiscale parameter $v$ is related to the fractal dimension $(v=5-2 D$ for mono-dimensional Euclidean surfaces and $v=7-2 \mathrm{D}$ for bidimensional surfaces [7]) and $\gamma$ is related to the standard deviation and the number of spatial scales is equal to P. In a previous work [4][5], to describe more adequately natural surfaced, we have used the separable dyadic multi-resolution analysis introduced by Mallat [8] to extend the wavelet theory from one-dimensional to two-dimensional case.

Using the bi-dimensional wavelet transform, we have obtained respectively the vertical wavelet component, the horizontal wavelet component (3) and the diagonal wavelet component (4) of the height $Z_{p}^{i}$, (where $\mathrm{i}=$ Vertical, Horizontal or Diagonal.

$$
\begin{aligned}
& Z_{p}^{V}(x, y)=\sum_{m_{x}=0}^{p} \sum_{m_{y}=0}^{p} \sum_{n_{x}=-\infty}^{+\infty} \sum_{n_{y}=-\infty}^{+\infty} Z_{n_{x}}^{m_{x}} Z_{n_{y}}^{m_{y}} \Psi\left(\frac{2^{m_{x}}}{B} x-n_{x}\right) \varphi\left(\frac{2^{m_{y}}}{B} y-n_{y}\right) \\
& Z_{p}^{H}(x, y)=\sum_{m_{x}=0}^{p} \sum_{m_{y}=0}^{p} \sum_{n_{x}=-\infty}^{+\infty} \sum_{n_{y}=-\infty}^{+\infty} Z_{n_{x}}^{m_{x}} Z_{n_{y}}^{m_{y}} \varphi\left(\frac{2^{m_{x}}}{B} x-n_{x}\right) \Psi\left(\frac{2^{m_{y}}}{B} y-n_{y}\right) \\
& Z_{p}^{D}(x, y)=\sum_{m_{x}=0}^{p} \sum_{m_{y}=0}^{p} \sum_{n_{x}=-\infty}^{+\infty} \sum_{n_{y}=-\infty}^{+\infty} Z_{n_{x}}^{m_{x}} Z_{n_{y}}^{m_{y}} \Psi\left(\frac{2^{m_{x}}}{B} x-n_{x}\right) \Psi\left(\frac{2^{m_{y}}}{B} y-n_{y}\right)
\end{aligned}
$$

Their autocorrelation function (ACF) is given by:

$\rho^{i}(x, y, x+\xi, y+\eta)=\left\langle Z_{p}^{i}(x, y) Z_{p}^{i}(x+\xi, y+\eta)\right\rangle$

And the standard deviation can be written as:

$s^{2}=r_{c}^{H}(0,0)=r_{c}^{D}(0,0)=r_{c}^{V}(0,0)$

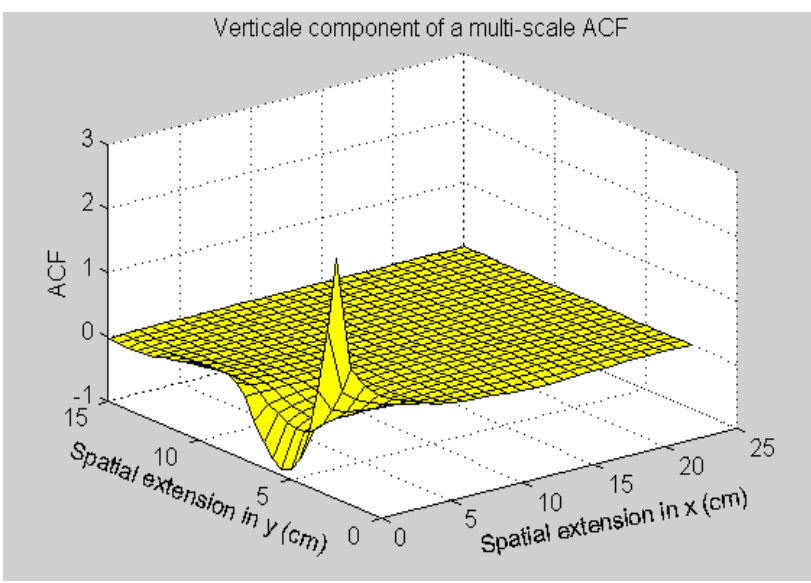

Figure 1. Vertical component of a MLS two-dimensional ACF for $v x=2.1 ; v y=1.1, \gamma x=0.2 \mathrm{~cm}$ and $\gamma y=0.8 \mathrm{~cm}$

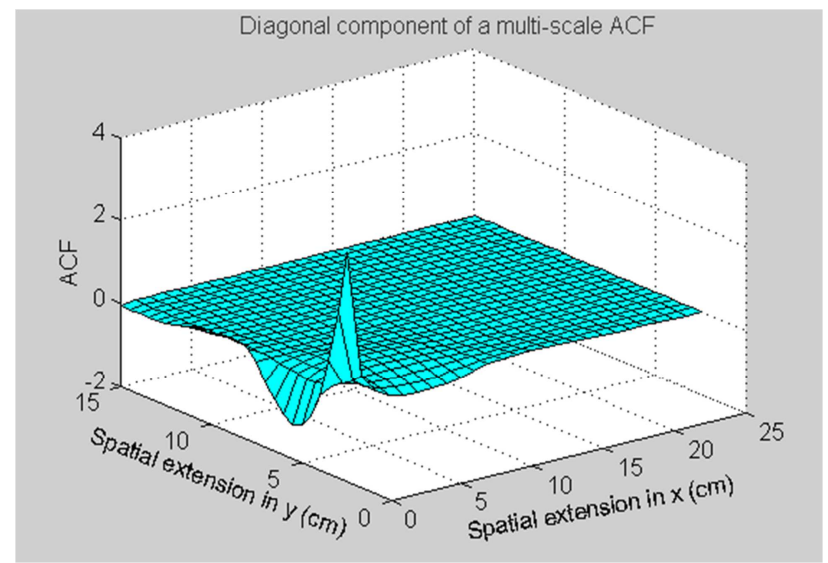

Figure 2. Diagonal component of a MLS two-dimensional ACF for $v_{x}=2.1 ; v_{y}=1.1, \gamma x=0.2 \mathrm{~cm}$ and $\gamma y=0.8 \mathrm{~cm}$

In figure 1 and figure 2 the vertical, diagonal and horizontal component of a multi-scale two dimensional ACF surface are represented.

We have simulated the 3D representation of the MLS surfaces for two different spatial scales, with $\mathrm{P}=5$ in figure 3 and $\mathrm{P}=10$ in figure 4 .

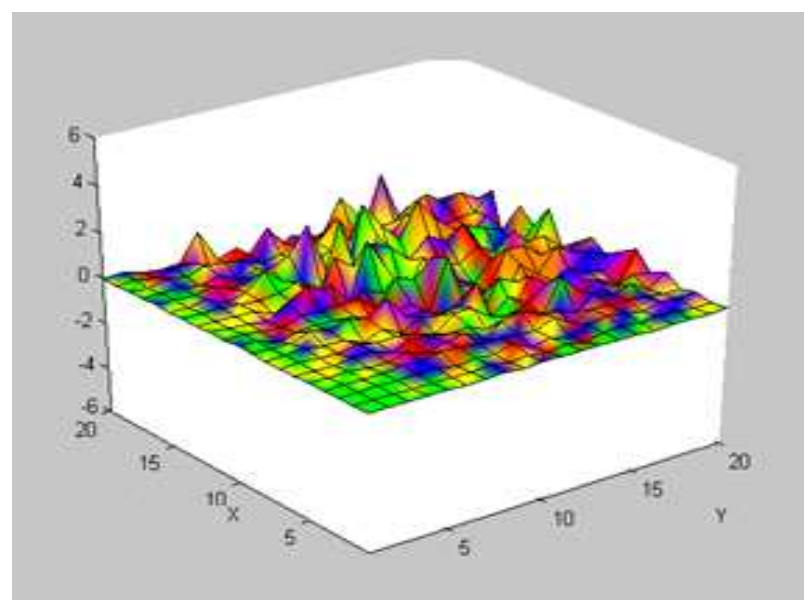

Figure 3. 3D representation of a multi-scale surface using Daubechies wavelet with multi-scale parameters $\left(v_{1}=1.3 ; v_{2}=1.3 ; \gamma=0.2 \mathrm{~cm}\right) P=5$ 


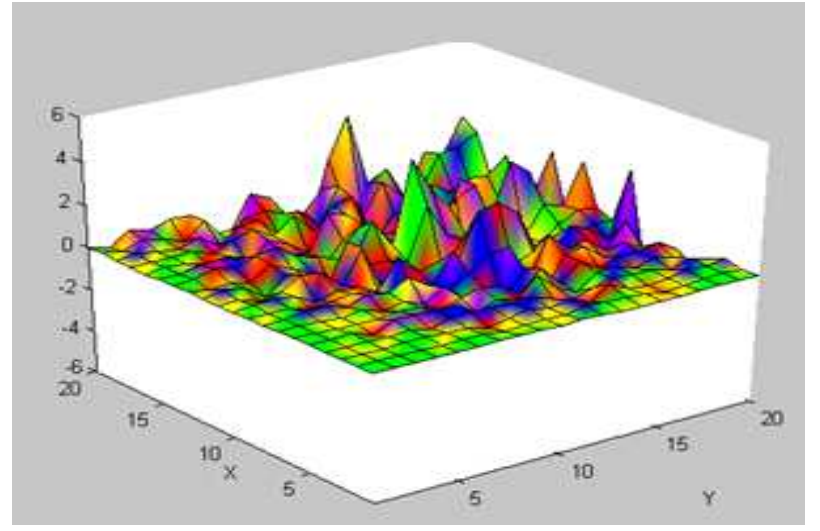

Figure 4. 3D representation of a multi-scale surface using Daubechies wavelet with multi-scale parameters $\left(v_{1}=1.3 ; v_{2}=1.3 ; \gamma=0.2 \mathrm{~cm}\right) P=10$

\section{A MULTILAYER MULTISCALE BI-DIMENSIONAL SPM MODEL}

\subsection{MLS SPM Model}

In this study, we modeled radar backscattering over a three layer model [10] by taking into account volume scattering. Each layer is described as a multi-scale bi-dimensional surface using our multi-scale description.

In this study the small perturbation model SPM is used for the simulation of backscattering coefficients.

SPM input parameters are the dielectric constant (deduced from the surface volumetric moisture content), the fractal parameter and the standard deviation of surface height. A multi-scale correlation function was therefore used in thisstudy. To remain within the domain of validity of the SPM used surfaces with $\mathrm{ks}<0.3$ (k: wave number, $\mathrm{s}$ : rms height).

$\sigma^{0}=\frac{k}{4} \exp \left(-2 k^{2}\right)\left(\cos ^{2} \theta\right) s^{2}\left|I_{q p}\right| W(-2 k \sin \theta, 0)$

Where $\Theta$ is the incident angle and $\left|I_{q p}\right|$ is given by given by Fung [6] and

$W^{(n)}\left(-2 k_{x}, 0\right)=\frac{2}{\pi} \int_{0}^{\infty} \int_{0}^{\infty}\left(\frac{r_{c}^{i}(\xi, \eta)}{r_{C}^{i}(0,0)}\right)^{n} \cos \left(2 k_{x} \xi\right) d \xi d \eta$

Where $\mathrm{W}^{(\mathrm{n})}$ is the Fourier transform of the nth power of the multi-scale autocorrelation function given by Mattia in [7] with $\mathrm{n}=1$ for the SPM model [3][4][5][7]. Surfaces are characterized by the dielectric constant related to soil moisture, the albedo, the optical depth and surface roughness. Previous works used classical statistical parameters namely correlation length and standard deviation in the expression of the autocorrelation function $\mathrm{W}$. The principal aim of this study is to use the multiscale surface description in the backscattering coefficient.

\subsection{Multilayer modified SPM model}

In this section we present the multilayer reflection model given by Fung [6] and Song [10] using our multi-scale 2D description of surface roughness.
The natural soil is composed by a dense media composed by multiple species of particles [10] and water, of a discrete dielectric soil componente.

We considered the half-space below the ground surface $(\mathrm{z}<0)$ as a three-layer medium (figure 5), where $\mathrm{D}$ is the radar penetration depth. This multilayer soil model includes three uniform layers [10]:

- $\quad$ The medium 1 with thickness $d_{1}$ and permittivity $\varepsilon_{s w}$ represents the mixture of soil particles and liquid water contents;

- The medium 2 with thickness $\mathrm{d}_{2}$ and permittivity $\varepsilon_{0}$ represents the air in soil;

The medium 3 represents the soil layer below the radar penetration depth $\mathrm{D}\left(\mathrm{d}_{1+} d_{2}\right)$, with permittivity $\varepsilon_{\mathrm{r}}$. It is semiinfinite and has no thickness.

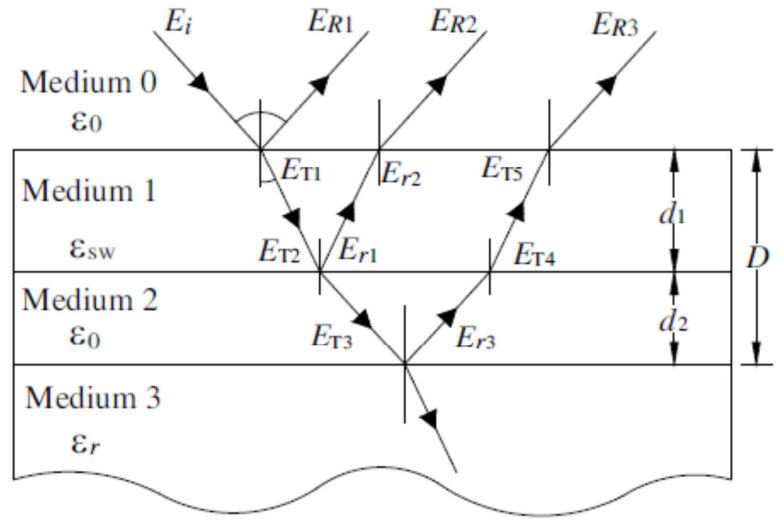

Figure 5. Multilayer reflection model soil [10]

We consider the reflection of an electromagnetic wave from each layer. The incident wave is from layer1, and as layer 3 is semi-infinite there is no electromagnetic wave reflection from the bottom of layer3.

The incidence and reflected radar signal between medium 0 (air) and medium 1 can be expressed as:

$E_{i}=E_{0} e^{-j k_{1 Z^{Z}}}$

$E_{R 1}=R_{a} E_{0} e^{-j K_{z} z}=R_{a} E_{i}$

$E_{R 2}=T_{10} A R_{S} A T_{01} E_{i}=T_{10} T_{01} A^{2} R_{S} E_{i}$

$E_{R a}=T_{10} A T_{21} R_{a} T_{12} \mathrm{~A} T_{01}$

$E i=T_{01} T_{12} T_{21} A^{2} R_{a} E_{i}$

We have to take into account these equalities since medium 2 is air:

$R_{s}=-R_{a}$

$T_{01}=T_{21}$

$T_{12}=T_{10}$ 
The total surface reflection coefficient of the multilayer soil can be expressed from the incident and reflected radar signal at airmedium 1 interface as

$\tilde{R}_{a}=R_{a}+R_{a} T_{01} T_{10} A^{2}\left(T_{01} T_{10}+1\right)$

where $R_{a}$ is the surface scattering of the soil (the specular surface reflection term), $R_{a} T_{01} T_{10} A^{2}\left(T_{01} T_{10}+1\right)$ is the internal reflections between layers (the equivalent volumetric scattering term), with $\mathrm{A}=e^{\frac{k_{e} d_{1}}{\cos \theta_{t}}}, \theta_{t}$ the refraction angle at the air medium 1 interface, $R_{a}$ the specular reflection coefficient of air at airmedium, $T_{m n}$ the transmission coefficient from medium $\mathrm{m}$ to medium $\mathrm{n}(\mathrm{n}=0,1,2,3), K_{e}$ the extinction coefficient of the medium 1 ([7], [10]) $d_{1}$ the thickness of the medium 1 .

The total reflection coefficients of the natural soil are computed using the multilayer model, and volumetric scattering is approximated by the internal reflections between layers. The surface reflection terms in the modified SPM model are replaced by the total reflection coefficients from the multilayer soil surface. The original multi-scale SPM model includes only the surface scattering of the natural bare soil, while the multilayer soil modified 2D MLS SPM model includes both the surface scattering and the volumetric scattering within the soil. This multilayered model has been used to calculate the total surface reflection coefficients of a natural soil surface for both horizontal and vertical co-polarizations.

Each layer is described as a multi-scale bi dimensional surface using our multi-scale description ([4] [5] [10]) and the modified SPM.

\section{SENSITIVITY ANALYSIS OF THE THREE LAYERS MULTISCALE BI-DIMENSIONAL SPM MODEL}

\subsection{Sensitivity to multi-scale roughness parameters}

We have considered the VV and $\mathrm{HH}$ polarizations and studied the sensitivity of radar backscattering and angular trends for different multi-scale roughness and for different dielectric constants of each layer.

We have simulated the angular trends of the three layers multiscale backscattering coefficient from 20 to 80 degrees for different roughness parameters.

As a first step, we fixed the parameter related to the Root Mean Square at $0.0031 \mathrm{~cm}$ in VV and $\mathrm{HH}$ polarizations for five spatial scales to find out the effect of fractal dimension on the radar backscattered signal (Figure 6 and figure 7).

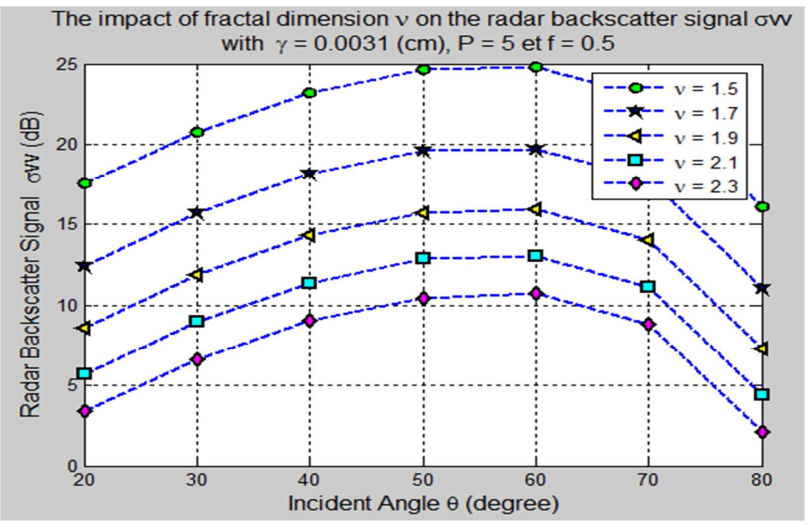

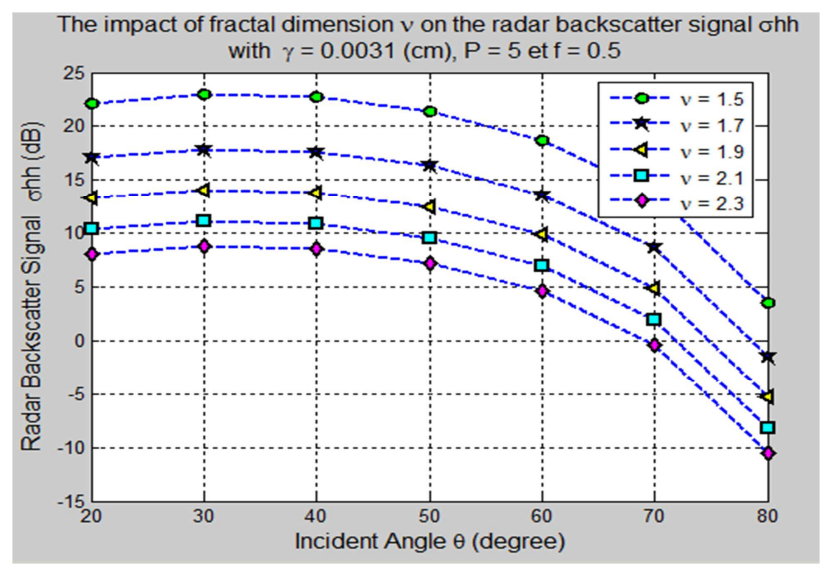

Figure 7. Backscattering coefficient dependence on fractal parameter $\mathrm{v}$ at $\mathrm{HH}$ polarization

When $v$, the parameter related to the fractal dimension, increases the backscattering coefficient decreases.

As surfaces with $v$ between 1.5 and 2.3 are considered as smooth, we set, as a second step, this parameter at 2.1 in VV polarization and 1.9 in $\mathrm{HH}$ polarization for five spatial scales (Figure 8 and figure 9).

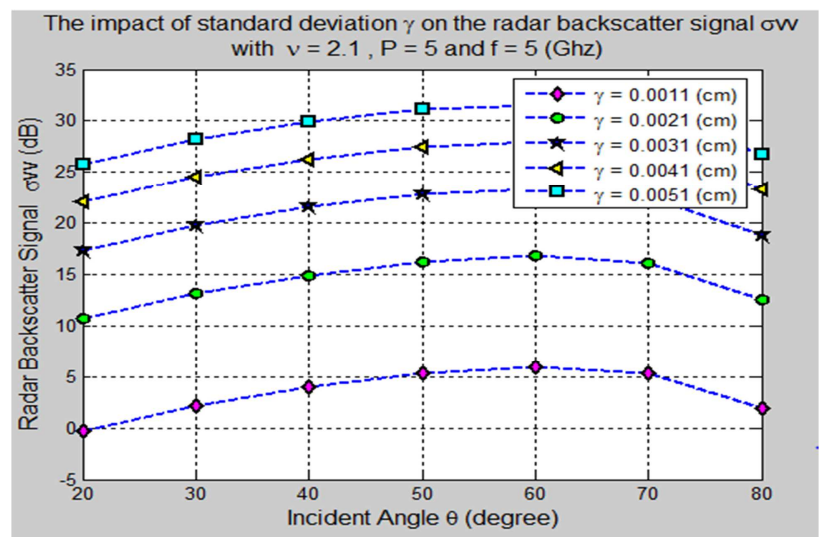

Figure 8. Backscattering coefficient dependence on standard deviation $\gamma$ at $\mathrm{VV}$ polarization

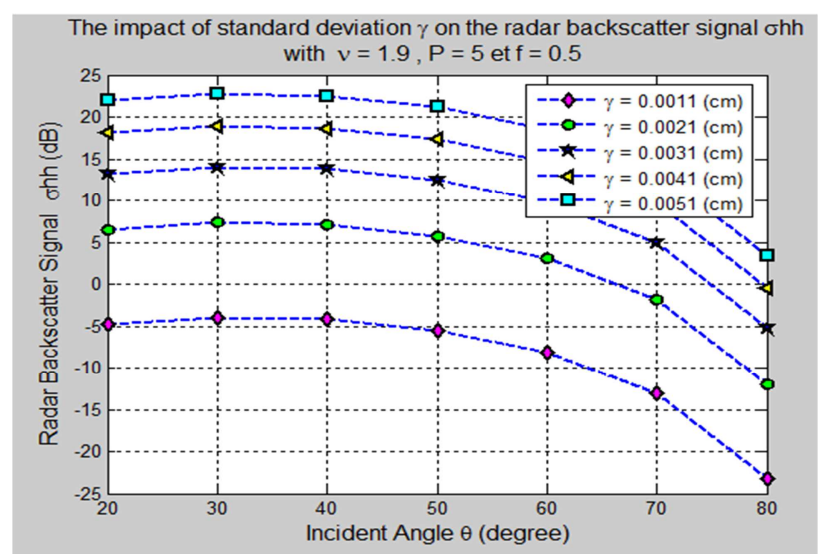

Figure 9. Backscattering coefficient dependence on standard deviation $\gamma$ at $\mathrm{HH}$ polarization

Figure 6. Backscattering coefficient angular trends on fractal parameter $v$ at $\mathrm{VV}$ polarization 
When $\gamma$, the parameter related to the fractal dimension, increases the backscattering coefficient increases.

The backscattered signal in VV polarization is higher than the backscattered signal in $\mathrm{HH}$ polarization.

For all the simulations, the backscattering coefficient decreases with the incidence angle.

\subsection{Sensitivity to Soil Moisture}

Soil moisture is related to the complex dielectric constant $\varepsilon$. In Figure 10, figure 11, figure 12 and figure 13, we have represented radar backscattering as angular trends for different values of the complex permittivity of the second layer in the two polarizations $\mathrm{VV}$ and $\mathrm{HH}$.

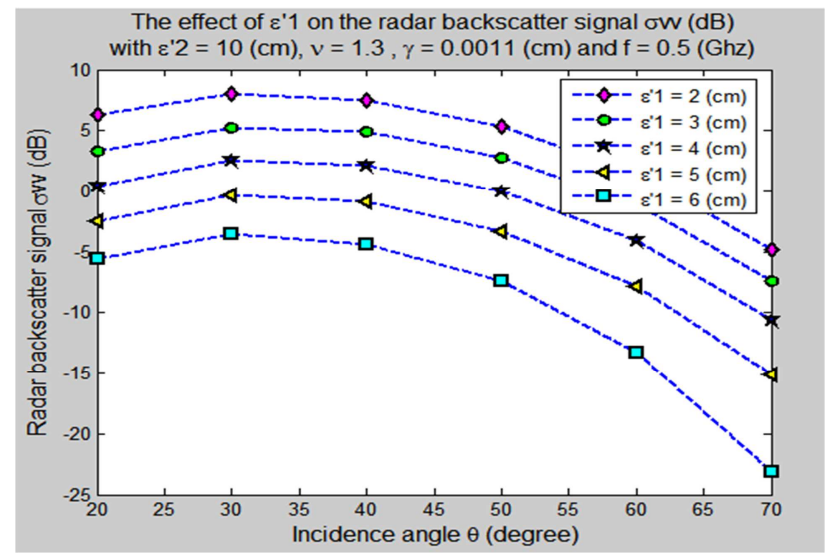

Figure 10. Backscattering coefficient dependence on $\varepsilon$ '1 at VV polarization

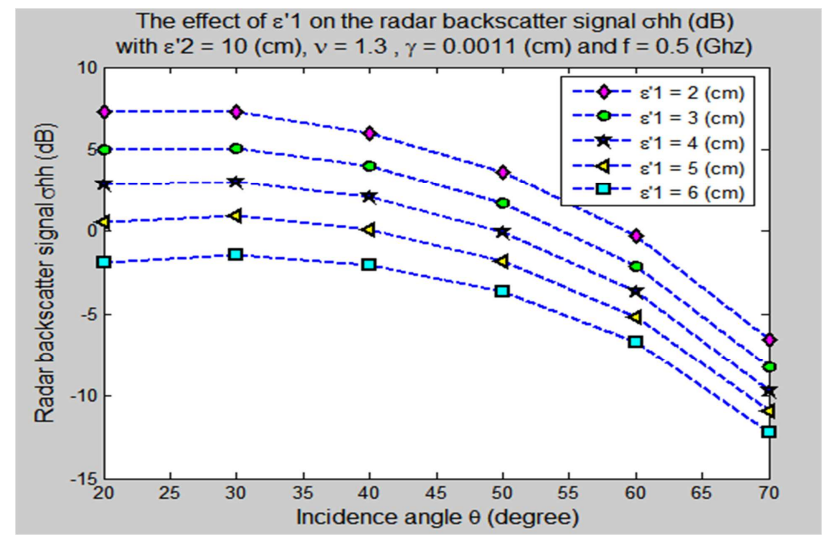

Figure 11. Backscattering coefficient dependence on $\varepsilon$ '1 at HH polarization

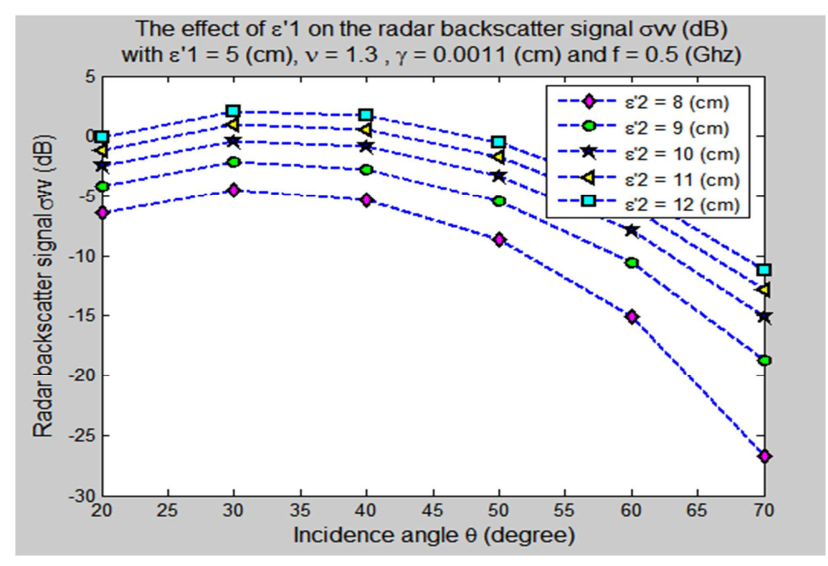

Figure 12.Backscattering coefficient dependence on $\varepsilon_{2}$ at VV polarization

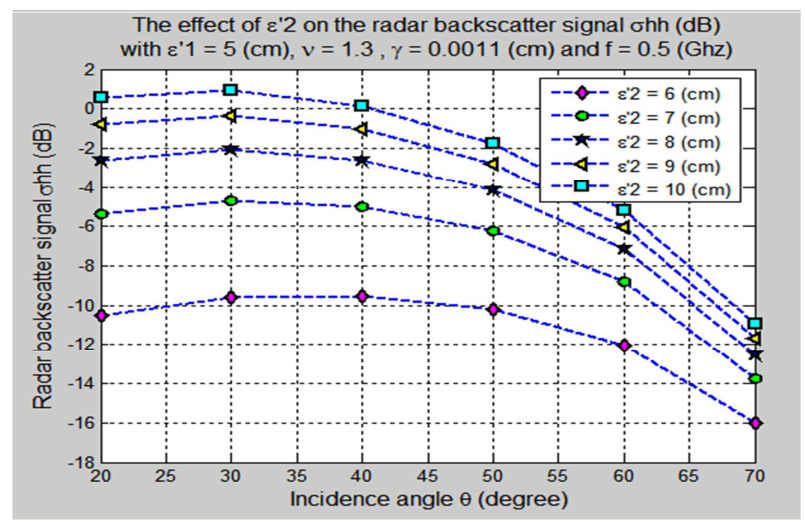

Figure 13. Backscattering coefficient dependence on $\varepsilon_{2}^{\prime}$ at HH polarization

The backscattering coefficient decreases as $\varepsilon^{\prime}{ }_{1}$ increases, whereas it increases where $\varepsilon^{\prime}{ }_{1}$ increases also. Indeed when the layers are dry corresponding to a lower humidity and as a consequence a lower dielectric constant, the penetration of the signal is more important and the backscattered signal is lower. As the dielectric constant increases, the surfaces and subsurface become wetter and the backscattered signal increases because the penetration is lower.

\section{METHODOLOGY OF THE RETRIEVAL PROCEDURE}

\subsection{Inversion procedure}

We present in this section, an algorithm to retrieve multi-scale roughness parameters and soil moisture parameter. In this study, the direct problem is represented by the SPM model. Thus, a sensitivity analysis of the SPM model has been performed and presented in the section 5.3 to examine the dependence of the output of the scattering model to the inputs parameters. When the outputs of the scattering model became saturated or insensitive to a parameter, the parameter inversion range was narrowed.

The method consists of inverting the SPM direct model using multilayer perceptron architecture [4] and [6]. The inversion consists in retrieving roughness and soil moisture parameters $\gamma 1, \gamma 2, v 1, v 2, \varepsilon 1$ et $\varepsilon 2$ by using as input parameters the radar backscattering coefficients $\sigma \mathrm{HH}, \sigma \mathrm{VV}$ and the incident angle 
$\theta$ varied from 30 to 60 degrees. The NN is trained by learning rules using the back propagation method.

Simulated data sets based on the SPM surface scattering model are used to train the neural network.

To illustrate the inversion techniques we propose a methodology given by figure 14 .

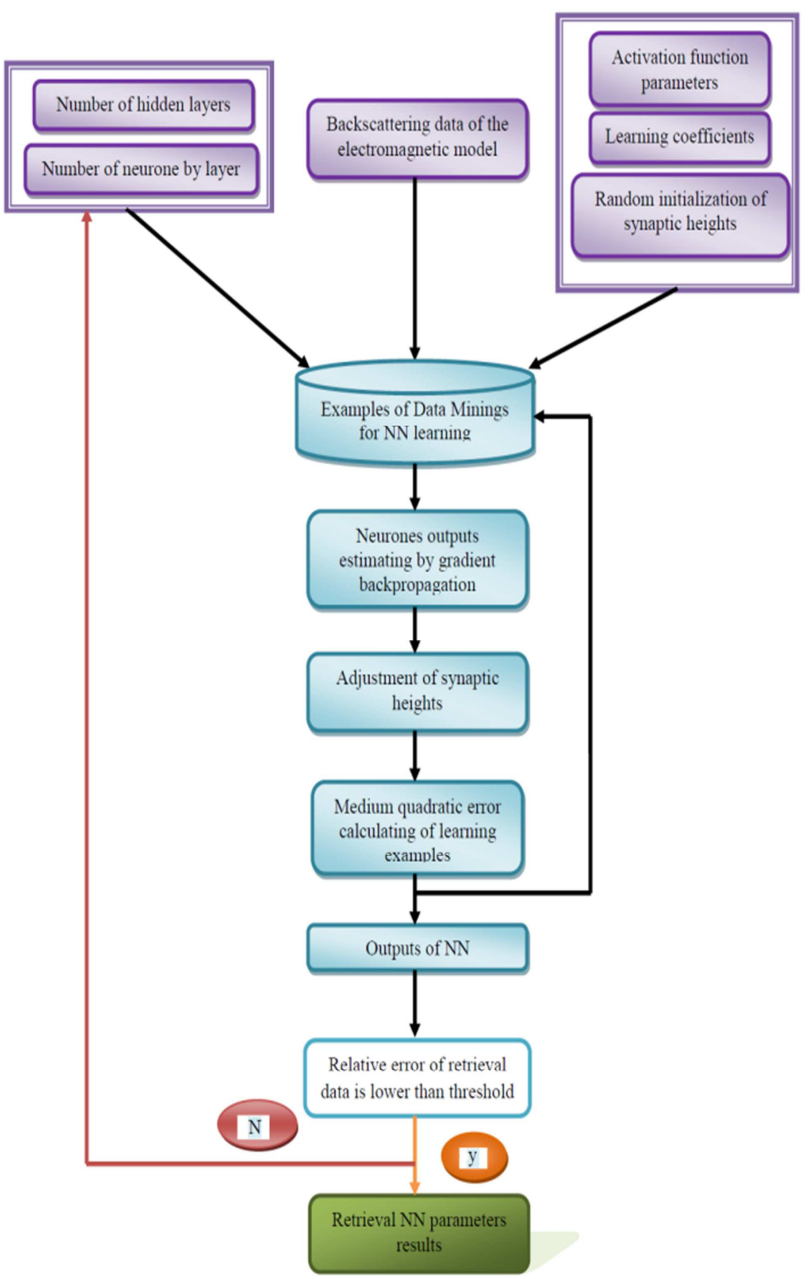

Figure 14. The proposed methodology

\subsection{Neural Network Training}

The first step in the inversion procedure is the generation of a set of training patterns.

In this study, a total of 35390 training patterns were generated by using each of the signal models $\sigma$ of the SPM backscattering coefficient. The parameters of interest $\sigma$ used to generate the training patterns were randomly selected from within the range of parameters given by the sensitivity analysis.

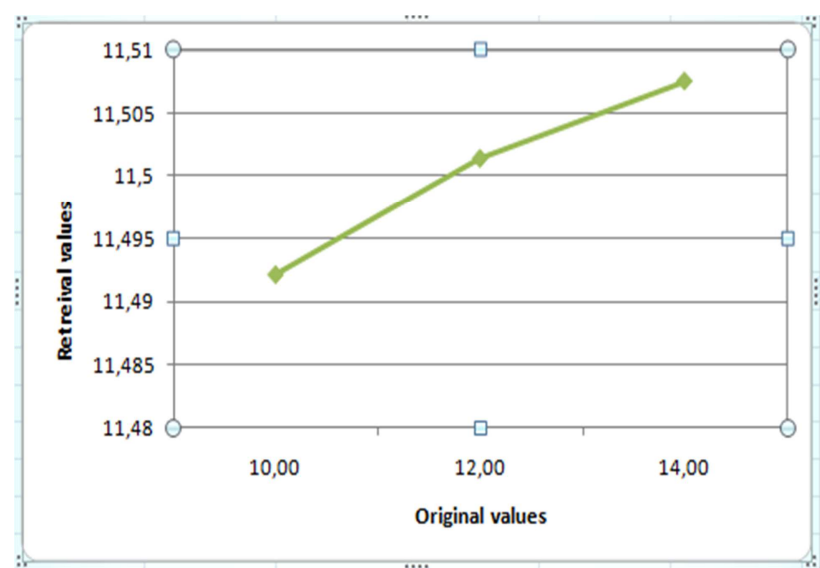

Figure 15. The retrieving soil moisture parameter $\varepsilon_{1}$ after the inversion by the $\mathrm{NN}$ function of its original value

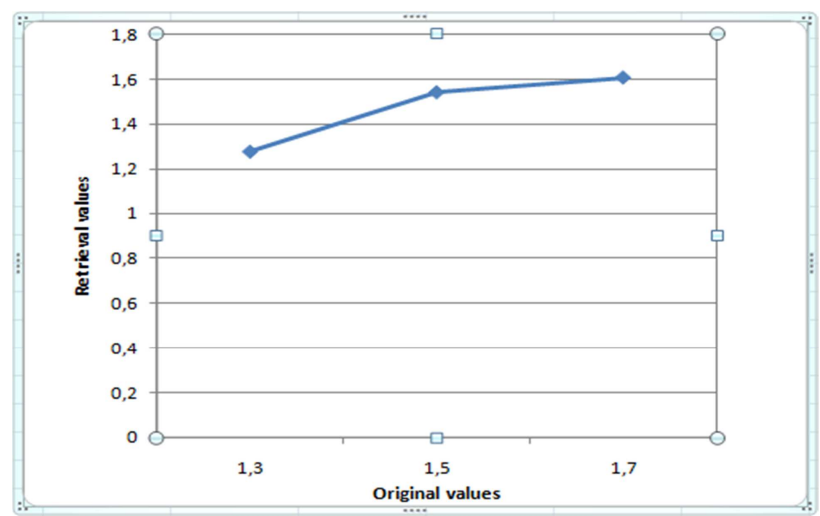

Figure 16. The retrieving roughness parameter $\gamma_{1}$ after the inversion by the $\mathrm{NN}$ function of its original value

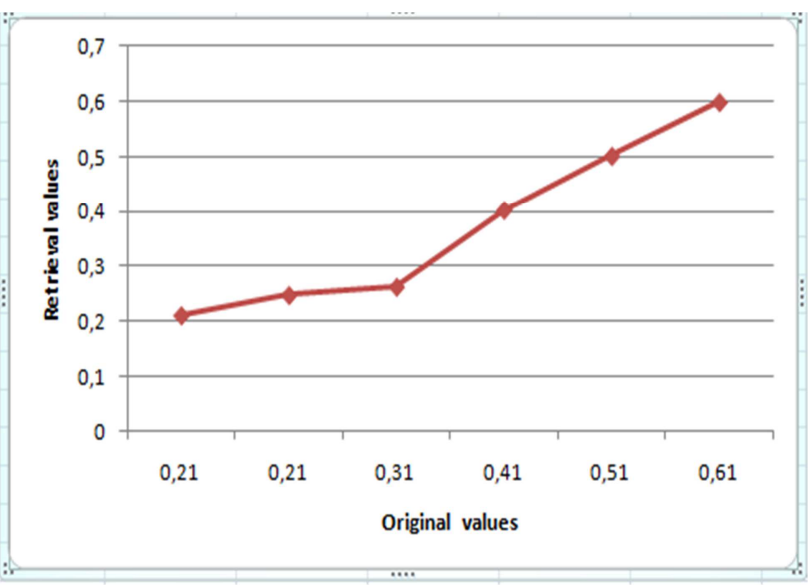

Figure 17. The retrieving roughness parameter $v_{1}$ after the inversion by the $\mathrm{NN}$ function of its original value

\subsection{Inversion Algorithm Results}

To illustrate the inversion techniques described in the previous section, we apply them to the data simulated by the SPM.

Before using the $\mathrm{NN}$ for the inversion, we have to calculate the mean rms error of the network. It converges well to a value smaller than 0.05 after 6000 iterations so that the $\mathrm{NN}$ is ready for the inversion procedure. 
In figure 18 we present the sum squared network error for 35390 epochs. The inversion has given quite satisfactory results as the original values were retrieved with an error of $2.75 \%$.



Figure 18. The sum squared network error

\section{CONCLUSION}

In this paper, we have presented a lossy 2D multi-scale multilayered medium backscattering model.

We replaced the surface reflection coefficients in the SPM model, which was obtained from a single-layer model, by the total reflection coefficients obtained from the three layered model. We have studied angular trends and three layers MLS SPM backscattering behavior in both $\mathrm{HH}$ and VV polarization for different roughness and dielectric parameters.

After this sensitivity study we performed the inversion using a neural network technique witch leaded to quite satisfactory results with a mean error of $2.75 \%$.

Future work will be dedicated to the study of radar backscattering on $n$ layered media.

\section{REFERENCES}

\section{References from Books:}

[1] Ulaby, F.T., Moore, M.K. and Fung A.K. "Microwave remote sensing Active and passive" Artech House, Norwood, M.A., 3, (1986).

\section{References from Other Literature:}

[2] Chen, M.F. and Fung, A.K. "A numerical study of the regions of validity of the Kirchhoff and Small Perturbations rough scattering models", Papers, IEEE Transaction On Geosciences And Remote Sensing 23(2), 136-170 (1988).

[3] Davidson, M., Le Toan, T., Mattia, F., Satalino, G., Manninen, T. and Borgeaud, M. "On the characterization of agricultural soil roughness for radar sensing studies", Papers, IEEE Transaction on Geosciences and Remote Sensing, Papers $38,630-640$ (2000).

[4] Farah, L. B, Farah, I. R., Bennaceur, R., Belhadj, Z. and Boussema, M. R. "Soil multiscale roughness parameters and soil moisture retrieval from radar backscattering using a neural network technique," Proc.
PIERS, Progress In Electromagnetic Research Symposium, Tokyo,(2006).

[5] Farah, L. B, Farah, I. R., Bennaceur, R, Hosni, I. and Boussema, M. R. "A Two Layers Multiscale Bi-dimensional SPM Model for the Study of Radar Backscatter Behavior on Semi-arid Soil Subsurfaces" Papers, PIERS ONLINE, Progress In Electromagnetic Research, 6 (2010).

[6] Fung, A.K. "Microwave scattering and emission models and their applications" Artech House, (1994).

[7] Mattia, F. and Le Toan, T. "Backscattering properties of multiscale rough surfaces," Journal of Electromagnetic Waves and Applications, Papers 13(4), 493-528 (1999).

[8] Mallat, S.G. "Theory of multi-resolution signal decomposition: The Wavelet representation". Papers, IEEE Transactions on Pattern analysis and machine intelligence, 11(7), 674-693 (1989).

[9] Oh, Y., Sarabandi, K. and Ulaby, T. "An empirical model and an inversion technique for radar scattering from bare soil surfaces", Papers, IEEE Transactions on Geosciences and Remote Sensing, 30, 370-381 (1992).

[10] Song. K, X. Zhou and Fan,Y. "Multilayer soil model for improvement of soil moisture estimation using the small perturbation method", Papers, Journal of Applied Remote Sensing, 3, 033567 (2009).

[11] Zribi, M., Le Morvan, A. and Baghdadi, N. "Dielectric Constant Modelling with Soil-Air Composition and Its Effect on Sar Radar Signal Backscattered over Soil Surface", Papers, Sensors, 8, 6810-6824 (2008). 